Copyright by the American Physical Society. Tackett, A. R.; Di Ventra, M., "Targeting specific eigenvectors and eigenvalues of a given Hamiltonian using arbitrary selection criteria," Phys. Rev. B 66, 245104 DOI: http://dx.doi.org/10.1103/

\title{
Targeting specific eigenvectors and eigenvalues of a given Hamiltonian using arbitrary selection criteria
}

\author{
Alan R. Tackett \\ Department of Physics and Astronomy, Vanderbilt University, Nashville, Tennessee 37235 \\ Massimiliano Di Ventra \\ Department of Physics and Center for Self-Assembled Nanostructures and Devices, Virginia Polytechnic Institute and State University, \\ Blacksburg, Virginia 24061-0435
}

(Received 9 May 2002; revised manuscript received 1 July 2002; published 5 December 2002)

\begin{abstract}
We present a method for calculating some select eigenvalues and corresponding eigenvectors of a given Hamiltonian. We show that it is possible to target the eigenvalues and eigenvectors of interest without diagonalizing the full Hamiltonian, by using any arbitrary physical property of the eigenvectors. This allows us to target, for example, the eigenvectors based on their localization properties (e.g., states localized at a given surface or interface). We also show that the method scales linearly with system size.
\end{abstract}

DOI: $10.1103 /$ PhysRevB.66.245104

PACS number(s): 71.15.-m, 31.15.Ct, 31.15.Ew

\section{INTRODUCTION}

The calculation of the eigenvalues and corresponding eigenvectors of a given Hamiltonian $H$ is of fundamental importance in quantum mechanics. In many physical problems, it is enough to determine the eigensolutions of $H$ that correspond to the lowest-energy states of the spectrum. In this case, several numerical methods are available that efficiently allow us to diagonalize $H$ with respect to the lowestenergy states. ${ }^{1-5}$ However, there are many physics problems that require knowledge of only some select eigenvalues and corresponding eigenvectors of $H$, which are not the lowestenergy states of the spectrum, and for which the diagonalization of the full Hamiltonian is computationally very expensive if not impossible. ${ }^{6-8}$ For these problems, the determination of such eigensolutions presents a challenge. To this end, Wang and Zunger ${ }^{6}$ developed a method [the socalled "folded spectrum (FS)" method] which scales linearly with system size rather than the usual cubic scaling required by traditional matrix diagonalization techniques. ${ }^{9}$ The method consists of "folding" the eigenvalues of the spectrum around a given reference energy and "squaring" the resulting Hamiltonian operator. ${ }^{6}$ Using Hamiltonians constructed from semiempirical pseudopotentials, those authors have successfully applied the technique to a number of interesting problems including the calculation of the dielectric properties of quantum dots, ${ }^{7}$ variation of the band gap with quantum dot size, and the solution of the "inverse bandstructure problem." ${ }^{\prime 8}$ In all of these cases only a relatively small fraction of the total number of eigensolutions of the Schrödinger equation was determined around a specific energy. However, the "squaring" of the Hamiltonian operator in the FS method greatly increases the difficulty in solving the original eigenvalue problem. Moreover, in electronic structure calculations, the solution of a generalized eigenvalue problem is sometimes required. ${ }^{11,12}$ The FS method could, in principle, be extended to such cases but at the cost of a significant increase in solving difficulty. In addition, the FS method can only handle reference energies as selection criterion. However, in certain physical problems selection criteria other than the eigenenergies are more relevant to target specific eigensolutions of a given Hamiltonian, e.g., the localization properties of the wave functions at surfaces or interfaces.

We present in this paper an alternative approach to target select eigenvalues and corresponding eigenvectors by using arbitrary physical properties of the eigensolutions. The method relies on the use of the Jacobi-Davidson technique ${ }^{10}$ that does not require "squaring" the Hamiltonian operator as in the FS method, but solves the eigenvalue equation directly. This technique does not suffer from either of the FS problems and can easily be extended to generalized eigenvalue problems resulting from the use of particular methods and technicalities for the solution of the Schrödinger equation, such as the projector augmented wave (PAW) method ${ }^{11}$ or the use of ultrasoft pseudopotentials ${ }^{12}$ or a nonorthogonal basis set.

The paper is organized as follows. In Sec. II, we will briefly outline the problem we want to address and its current solution within the FS method. In Secs. III and IV, we present the Jacobi-Davidson method that represents the core of the alternative approach we propose. In Sec. V its practical implementation is outlined. In Sec. VI, we discuss its convergence properties and scalability with system size. Finally, in Sec. VII, we discuss few examples of application of the present approach where different selection criteria are used.

\section{THE EIGENVALUE PROBLEM}

The main concern in electronic structure calculations is solving the eigenvalue problem,

$$
\mathbf{H} \psi_{i}=\varepsilon_{i} \psi_{i},
$$

where $\psi_{i}$ 's are the electronic wave functions, $\mathbf{H}$ is the system Hamiltonian, and the $\varepsilon_{i}$ 's are the energy eigenvalues. Equation (1) can represent, for instance, a set of Kohn-Sham ${ }^{13}$ equations to determine the ground-state properties of a given system. A generalized eigenvalue equation can occur if a 
nonorthogonal basis set is chosen or as a result of the pseudopotential formalism. ${ }^{11,12}$ The generalized eigenvalue problem is defined as

$$
\mathbf{H} \psi_{i}=\varepsilon_{i} \mathbf{O} \psi_{i},
$$

where the new operator $\mathbf{O}$ is called the overlap operator. There are several techniques for trying to find a few of the smallest or largest eigenvalues, but few of them are effective in finding selected eigenvalues inside the spectrum. ${ }^{14}$ Furthermore, all of these methods rely on the eigenvalues as the selection criterion, and cannot be generalized to using other selection criteria.

\section{A. Folded spectrum method}

There are few methods for solving interior eigenvalue problems. ${ }^{22}$ The most successful approach to date is the folded spectrum method. ${ }^{6}$ This technique is based upon folding the eigenvalue spectrum around a reference energy $\varepsilon_{r e f}$, thus shifting the lowest eigenstate of the resulting system to that closest to the reference energy. The resulting eigenvalue equation is

$$
\left(\mathbf{H}-\varepsilon_{r e f}\right)^{2} \psi_{i}=\left(\varepsilon_{i}-\varepsilon_{r e f}\right)^{2} \psi_{i},
$$

which is then solved with standard techniques to find the lowest-energy states. ${ }^{6}$ The main disadvantage of this technique is that "squaring" the effective Hamiltonian operator also "squares" the condition number, which is directly related to the difficulty in solving the FS equation. This increase in the condition number makes solving the FS method much more difficult. As stated by the authors of the FS method, ${ }^{15}$ a typical case requires $\sim 100$ conjugate gradient (CG) steps per energy band per iteration. Each CG step requires two applications of $\mathbf{H}$ due to the squaring operation. This corresponds to $\sim 200$ applications of $\mathbf{H}$ per band per iteration. As the authors of FS method also point out, this is about the "square" of the normal number of CG steps required for solving the original eigenvalue equation. ${ }^{15}$

\section{B. Extending the FS method to generalized eigenvalue problems}

Even if never used in this context, the FS method can easily be extended to handle generalized eigenvalue problems. The resulting generalized folded spectrum equation is

$$
\left(\mathbf{H}-\boldsymbol{\varepsilon}_{r e f} \mathbf{O}\right) \mathbf{O}^{-1}\left(\mathbf{H}-\boldsymbol{\varepsilon}_{r e f} \mathbf{O}\right) \psi_{i}=\left(\varepsilon_{i}-\boldsymbol{\varepsilon}_{r e f}\right)^{2} \mathbf{O} \psi_{i} .
$$

However, from the above equation it is obvious that now the condition number and difficulty has increased "cubically" over the original eigenvalue problem, and we have implicitly assumed that the $\mathbf{O}$ operator can be inverted. ${ }^{16}$

\section{JACOBI-DAVIDSON METHOD}

The Jacobi-Davidson method, ${ }^{10}$ briefly described here, is an oblique projection method that solves the eigenvalue equation directly. This means that the condition number and difficulty in solving for the selected eigensolutions is the same as the original eigenvalue equation. As a result, han- dling generalized eigenvalue problems poses no difficulty. In the following, we refer to the latter case to illustrate the method.

The method consists of solving the projected eigenproblem with different search and test subspaces. The search subspace $\mathbf{V}=\left[\begin{array}{llll}\mathbf{v}_{1} & \mathbf{v}_{2} & \cdots & \mathbf{v}_{n}\end{array}\right]$ spans the space of the possible solutions, and the test subspace $\mathbf{W}=\left[\begin{array}{lll}\mathbf{w}_{1} & \mathbf{w}_{2} \cdots \mathbf{w}_{n}\end{array}\right]$ provides a space for testing the quality of the solutions. In most applications, the test and search subspaces are the same, leading to the following equation for the projected generalized eigenvalue problem:

$$
\mathbf{V}^{\dagger} \mathbf{H V} u_{i}=\varepsilon_{i} \mathbf{V}^{\dagger} \mathbf{O V} u_{i} .
$$

The new eigenvectors are then calculated as $\psi_{i}^{\text {new }}=\mathbf{V} u_{i}$. However, when targeting selected interior eigenvalues around some reference energy $\varepsilon_{r e f}$, it is more advantageous to make the test and search subspaces different. This leads to the following equation:

$$
\mathbf{W}^{\dagger} \mathbf{H V} u_{i}=\varepsilon_{i} \mathbf{W}^{\dagger} \mathbf{O V} u_{i} .
$$

If the test space is chosen as

$$
\mathbf{W}=\left(\mathbf{H}-\varepsilon_{r e f} \mathbf{O}\right) \mathbf{V},
$$

and $\mathbf{W}$ is made orthogonal, $\mathbf{W}^{\dagger} \mathbf{W}=\mathbf{I}$, the eigenvalue problem can be solved in an efficient way. First, let us shift the spectrum with the reference energy $\varepsilon_{r e f}$,

$$
\begin{gathered}
\mathbf{H} \psi_{i}=\varepsilon_{i} \mathbf{O} \psi_{i}, \\
\left(\mathbf{H}-\varepsilon_{r e f} \mathbf{O}\right) \psi_{i}=\left(\varepsilon_{i}-\varepsilon_{r e f}\right) \mathbf{O} \psi_{i}, \\
\widetilde{\mathbf{H}} \psi_{i}=\widetilde{\varepsilon}_{i} \mathbf{O} \psi_{i},
\end{gathered}
$$

where $\widetilde{\mathbf{H}}=\mathbf{H}-\boldsymbol{\varepsilon}_{r e f} \mathbf{O}$ and $\tilde{\varepsilon}_{i}=\varepsilon_{i}-\varepsilon_{r e f}$.

Now we can apply the oblique projection with $\mathbf{W}=\widetilde{\mathbf{H}} \mathbf{V}$ and $\psi_{i}=\mathbf{V} u_{i}$ to the eigenvalue equation giving

$$
\mathbf{W}^{\dagger} \widetilde{\mathbf{H}} \mathbf{V} u_{i}=\widetilde{\varepsilon}_{i} \mathbf{W}^{\dagger} \mathbf{O V} u_{i}
$$

Because $\mathbf{W}$ is orthogonal, we obtain

$$
\begin{gathered}
\mathbf{W}^{\dagger} \widetilde{\mathbf{H}} \mathbf{V} u_{i}=\tilde{\varepsilon}_{i} \mathbf{W}^{\dagger} \mathbf{O V} u_{i}, \\
\mathbf{W}^{\dagger} \mathbf{W} u_{i}=\widetilde{\varepsilon}_{i} \mathbf{W}^{\dagger} \mathbf{O V} u_{i}, \\
\mathbf{W}^{\dagger} \mathbf{O V} u_{i}=\frac{1}{\widetilde{\varepsilon}_{i}} u_{i}, \\
\mathbf{W}^{\dagger} \mathbf{O} \widetilde{\mathbf{H}}^{-1} \mathbf{W} u_{i}=\frac{1}{\tilde{\varepsilon}_{i}} \mathbf{u}_{i},
\end{gathered}
$$

since $\mathbf{V}=\widetilde{\mathbf{H}}^{-1} \mathbf{W}$.

Notice that this choice of the test subspace is mathematically equivalent to using a normal orthogonal projection method for computing the eigenvalues of

$$
\widetilde{\mathbf{H}}^{-1}=\left(\mathbf{H}-\boldsymbol{\varepsilon}_{r e f} \mathbf{O}\right)^{-1} .
$$




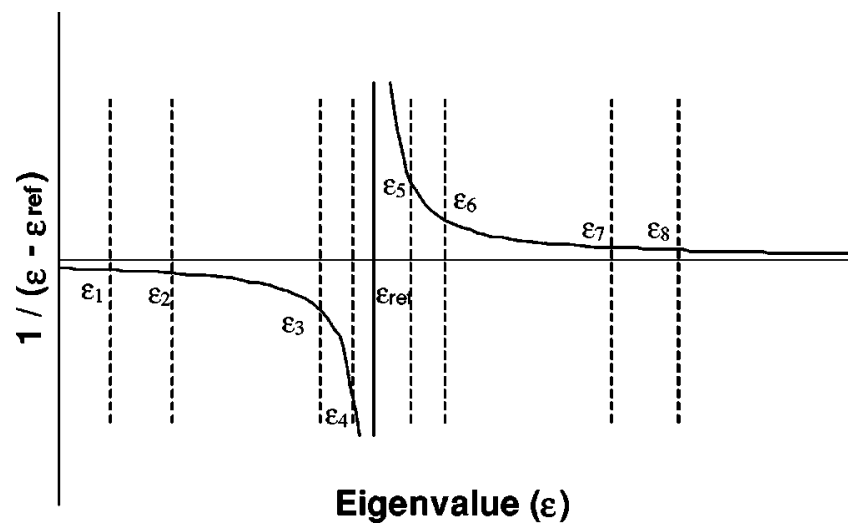

FIG. 1. Plot of the harmonic Ritz values vs original eigenvalue spectrum. The dashed lines correspond to the original eigenvalues with the solid vertical line in the center representing the reference energy chosen for targeting. The harmonic Ritz values are located at the intersection of the curved and dashed lines. Notice that original eigenvalues for states 4 and 5 are extrema of the harmonic Ritz problem.

However, no explicit inversion is necessary in the solution of Eq. (10). In this scheme the eigenvectors are the same as the original eigenvalue problem, but the eigenvalues are now shifted and inverted as

$$
\frac{1}{\varepsilon_{i}-\varepsilon_{r e f}}
$$

and are called harmonic Ritz values. ${ }^{10}$ This makes the original eigenvalues extremal eigenvalues of the shifted and inverted eigenproblem. This is illustrated in Fig. 1, where Eq. (12) is plotted as a function of the eigenvalue $\varepsilon$.

\section{PARTIAL GENERALIZED SCHUR FORM AND DEFLATION}

In the Jacobi-Davidson approach, one typically targets a single eigenvector and iterates until that eigenvector converges. In order to make sure that a converged eigenvector is not "found" again, it is important to be able to remove the converged eigenvectors from the eigenvalue problem. This is accomplished by deflating the original problem. If the test and search subspaces are the same, this is accomplished by simply projecting out the converged eigenvectors from the original problem. Let $\Psi=\left[\psi_{1} \psi_{2} \cdots \psi_{n}\right]$ where each column $\psi_{i}$ is a converged eigenvector. The deflated eigenproblem is then defined as

$$
\left(1-\mathbf{O} \Psi \Psi^{\dagger}\right)\left(\mathbf{H}-\varepsilon_{r e f} \mathbf{O}\right)\left(1-\Psi \Psi^{\dagger} \mathbf{O}^{\dagger}\right) \psi_{\text {new }}=0 .
$$

This requires memory for two vectors $\left(\psi_{i}\right.$ and $\left.\mathbf{O} \psi_{i}\right)$ for each converged eigenvector. A similar equation can be written if both the test and search subspaces are different but requiring four vectors for each converged eigenvector.

A better choice is to use a partial generalized Schur form that requires only two vectors per converged vector. The Schur vectors themselves are not eigenvectors of the eigenvalue equation, but it is easy to extract the eigenvectors from the Schur vectors. There is also a one-to-one correspondence between the converged Schur vectors and the actual eigenvectors.

A partial generalized Schur form for the matrix pair $(\mathbf{H}, \mathbf{O})$ is defined as

$$
\mathbf{H} \mathbf{Q}_{k}=\mathbf{Z}_{k} \mathbf{S}_{k}, \quad \mathbf{O} \mathbf{Q}_{k}=\mathbf{Z}_{k} \mathbf{T}_{k},
$$

where the matrices $\mathbf{Q}_{k}$ and $\mathbf{Z}_{k}$ are orthogonal $n \times k$ matrices, with $\mathbf{S}_{k}$ and $\mathbf{T}_{k}$ both being upper triangular matrices. Each column of $\mathbf{Q}_{k}$, denoted by $\mathbf{q}_{i}$, is a generalized Schur vector. The diagonal elements of $\mathbf{S}_{k}$ and $\mathbf{T}_{k}$ are related to the eigenvalues according to

$$
\varepsilon_{i}=\frac{S_{k}(i, i)}{T_{k}(i, i)} .
$$

The pair $\left(\mathbf{q}_{i},\left\langle S_{k}(i, i), T_{k}(i, i)\right\rangle\right)$ is referred to as a generalized Schur pair. Since $\mathbf{Q}_{k}$ and $\mathbf{Z}_{k}$ are both orthonormal, Eq. (14) can be rewritten as

$$
\mathbf{Z}_{k}^{\dagger} \mathbf{H} \mathbf{Q}_{k}=\mathbf{S}_{k}, \quad \mathbf{Z}_{k}^{\dagger} \mathbf{O} \mathbf{Q}_{k}=\mathbf{T}_{k} .
$$

Applying this to the original eigenvalue equation, we see it is easy to get the eigenvalues and eigenvectors from the partial generalized Schur form.

Let $\psi_{i}=\mathbf{Q}_{k} u_{i}$ then

$$
\begin{gathered}
\mathbf{H} \psi_{i}=\varepsilon_{i} \mathbf{O} \psi_{i}, \\
\mathbf{H} \mathbf{Q}_{k} u_{i}=\varepsilon_{i} \mathbf{O} \mathbf{Q}_{k} u_{i}, \\
\mathbf{Z}_{k}^{\dagger} \mathbf{H} \mathbf{Q}_{k} u_{i}=\varepsilon_{i} \mathbf{Z}_{k}^{\dagger} \mathbf{O} \mathbf{Q}_{k} u_{i}, \\
\mathbf{S}_{k} u_{i}=\varepsilon_{i} \mathbf{T}_{k} u_{i} .
\end{gathered}
$$

The eigenvectors are then calculated according to the original equation, $\psi_{i}=\mathbf{Q}_{k} u_{i}$.

The partial generalized Schur form can efficiently be used in conjunction with deflation. Assume that $\mathbf{Q}_{k}$ and $\mathbf{Z}_{k}$ currently exist and we seek to expand the generalized Schur form by finding suitable vectors $\mathbf{q}$ and $\mathbf{z}$ to calculate $\mathbf{Q}_{k+1}$ $=\left[\mathbf{Q}_{k} \mathbf{q}\right]$ and $\mathbf{Z}_{k+1}=\left[\mathbf{Z}_{k} \mathbf{z}\right]$. Then according to the definition, these vectors must satisfy

$$
\mathbf{H} \mathbf{Q}_{k+1}=\mathbf{Z}_{k+1} \mathbf{S}_{k+1}, \quad \mathbf{O} \mathbf{Q}_{k+1}=\mathbf{Z}_{k+1} \mathbf{T}_{k+1} .
$$

Substituting the above definitions into the previous equations gives

$$
\begin{aligned}
& \mathbf{H}\left[\mathbf{Q}_{k} \mathbf{q}\right]=\left[\mathbf{Z}_{k} \mathbf{z}\right]\left[\begin{array}{cc}
\mathbf{S}_{k} & \mathbf{s} \\
0 & \alpha
\end{array}\right], \\
& \mathbf{O}\left[\mathbf{Q}_{k} \mathbf{q}\right]=\left[\mathbf{Z}_{k} \mathbf{z}\right]\left[\begin{array}{cc}
\mathbf{T}_{k} & \mathbf{t} \\
0 & \beta
\end{array}\right] .
\end{aligned}
$$

We have introduced two new real numbers $\alpha$ and $\beta$, such that $\varepsilon=\alpha / \beta$. Expanding the above equations gives for the new columns 


$$
\begin{aligned}
& \mathbf{H q}=\mathbf{Z}_{k} \mathbf{s}+\mathbf{z} \alpha \rightarrow \mathbf{s}=\mathbf{Z}_{k}^{\dagger}(\mathbf{H q}-\mathbf{z} \alpha), \\
& \mathbf{O q}=\mathbf{Z}_{k} \mathbf{t}+\mathbf{z} \beta \rightarrow \mathbf{t}=\mathbf{Z}_{k}^{\dagger}(\mathbf{O q}-\mathbf{z} \beta) .
\end{aligned}
$$

Using the substitutions given above for $\mathbf{s}$ and $\mathbf{t}$, and combining the equations to eliminate the terms involving $\mathbf{z}$ gives

$$
\left(1-\mathbf{Z}_{k} \mathbf{Z}_{k}^{\dagger}\right)(\beta \mathbf{H}-\alpha \mathbf{O}) \mathbf{q}=0 .
$$

Note that the new vectors must also satisfy the orthonormal constraints $(\mathbf{q} \perp \mathbf{Q}$ and $\mathbf{z} \perp \mathbf{Z})$ and that the eigenvalues are defined as $\varepsilon=\alpha / \beta$, so that Eq. (21) can be expressed as

$$
\left(1-\mathbf{Z}_{k} \mathbf{Z}_{k}^{\dagger}\right)(\beta \mathbf{H}-\alpha \mathbf{O})\left(1-\mathbf{Q}_{k} \mathbf{Q}_{k}^{\dagger}\right) \mathbf{q}=0 .
$$

This means that the generalized Schur pairs are also solutions to the deflated eigenvalue problem. The eigenvectors can then be calculated from the Schur pairs by using Eq. (17) along with the definition $\psi_{i}=\mathbf{Q}_{k} u_{i}$.

\section{IMPLEMENTATION OF THE JACOBI-DAVIDSON METHOD}

In an actual implementation of the method, we start from a random guess for $\mathbf{q}$ and $\mathbf{z}$ with the $\mathbf{Q}, \mathbf{Z}, \mathbf{V}$, and $\mathbf{W}$ matrices set to null. $\mathbf{Q}$ and $\mathbf{Z}$ contain the partial generalized Schur form of the converged Schur vectors with $\mathbf{V}$ and $\mathbf{W}$ defining the search and test subspaces. The first step is to solve the Jacobi-Davidson equation for the correction vector $\mathbf{v}$, which will be used to augment the search and test subspaces. The Jacobi-Davidson equation is easily derived:

$$
\begin{gathered}
\left(\mathbf{H}-\varepsilon_{r e f} \mathbf{O}\right)(\mathbf{q}+\mathbf{v})=0, \\
\left(\mathbf{H}-\varepsilon_{r e f} \mathbf{O}\right) \mathbf{v}=-\left(\mathbf{H}-\boldsymbol{\varepsilon}_{r e f} \mathbf{O}\right) \mathbf{q}, \\
\left(\mathbf{H}-\boldsymbol{\varepsilon}_{r e f} \mathbf{O}\right) \mathbf{v}=-\mathbf{r},
\end{gathered}
$$

where $\mathbf{r}=\left(\mathbf{H}-\varepsilon_{\text {ref }} \mathbf{O}\right) \mathbf{q}$.

Since only the component of $\mathbf{v}$ orthogonal to $\mathbf{q}$ contains any new information, we can enforce this condition by removing this direction from both the test and search subspaces according to

$$
\left(1-\mathbf{z z}^{\dagger}\right)\left(\mathbf{H}-\varepsilon_{r e f} \mathbf{O}\right)\left(1-\mathbf{q q}^{\dagger}\right) \mathbf{v}=-\mathbf{r} .
$$

As stated earlier one also wants to ensure that already converged Schur vectors are not "revisited" by deflating the correction equation. The converged Schur vectors are stored in $\mathbf{Q}$ and $\mathbf{Z}$ as described earlier. Using this and the definition $\varepsilon_{r e f}=\alpha_{r e f} / \beta_{r e f}$, the general form for the Jacobi-Davidson correction equation is given by

$$
\begin{gathered}
\left(1-\mathbf{Z Z}^{\dagger}\right)\left(1-\mathbf{z Z}^{\dagger}\right)\left(\beta_{r e f} \mathbf{H}-\alpha_{r e f} \mathbf{O}\right) \\
\times\left(1-\mathbf{q q}^{\dagger}\right)\left(1-\mathbf{Q} \mathbf{Q}^{\dagger}\right) \mathbf{v}=-\mathbf{r} .
\end{gathered}
$$

This can also be combined with a preconditioner as discussed in Sec. 2.6 of Ref. 10. It is not necessary to solve Eq. (25) exactly. Typically just a few steps of an iterative method such as GMRES or BICGSTAB are required. ${ }^{17,18}$ The choice of $\alpha_{r e f}$ and $\beta_{r e f}$ is important and discussed in detail in Sec. 2.4 of Ref. 10 and given below

$$
\alpha_{r e f}=\frac{\varepsilon_{r e f}}{\sqrt{1+\varepsilon_{r e f}^{2}}}, \quad \beta_{r e f}=\frac{1}{\sqrt{1+\varepsilon_{r e f}^{2}}} .
$$

The next step is to update the test and search subspaces. Since we are dealing with the orthonormal matrices, the search subspace is updated as

$$
\begin{gathered}
\mathbf{v}_{\perp} \leftarrow \mathbf{v} \perp \mathbf{V}, \\
\mathbf{v}_{\perp} \leftarrow \mathbf{v}_{\perp} /\left\|\mathbf{v}_{\perp}\right\|, \\
\mathbf{V} \leftarrow\left[\mathbf{V} \mathbf{v}_{\perp}\right] .
\end{gathered}
$$

We recall that $\mathbf{v}$ is by definition orthogonal to $\mathbf{Q}$. The test subspace vector $\mathbf{w}$ is defined as

$$
\mathbf{w}=\left(\beta_{r e f} \mathbf{H}-\alpha_{r e f} \mathbf{O}\right) \mathbf{v} .
$$

As before, we are only interested in the component orthogonal to both $\mathbf{W}$ and $\mathbf{Z}$ :

$$
\begin{gathered}
\mathbf{w}_{\perp} \leftarrow \mathbf{w} \perp(\mathbf{W} \text { and } \mathbf{Z}), \\
\mathbf{w}_{\perp} \leftarrow \mathbf{w}_{\perp} /\left\|\mathbf{w}_{\perp}\right\|, \\
\mathbf{W} \leftarrow\left[\mathbf{W} \mathbf{w}_{\perp}\right] .
\end{gathered}
$$

The next step is to perform a subspace rotation by solving the projected generalized Schur problem for the matrix pair or

$$
\mathbf{U}_{L}^{\dagger}\left(\mathbf{W}^{\dagger} \mathbf{H V}\right) \mathbf{U}_{R}=\mathbf{S}, \quad \mathbf{U}_{L}^{\dagger}\left(\mathbf{W}^{\dagger} \mathbf{O V}\right) \mathbf{U}_{R}=\mathbf{T},
$$

for the left $\left(\mathbf{U}_{L}\right)$ and right $\left(\mathbf{U}_{R}\right)$ generalized Schur vectors and upper triangular matrices $\mathbf{S}$ and $\mathbf{T}$. Remember that the eigenvalues can be calculated with the diagonal elements of $\mathbf{S}$ and $\mathbf{T}$, according to Eq. (15).

\section{A. Arbitrary selection criteria}

The most obvious selection criterion is targeting a specific range of the eigenvalue spectrum. Another option consists of targeting the eigenvectors based on their localization property. In general, using the present approach, an arbitrary selection criterion can be used for selecting eigenvectors. This can be done in the following way. It is possible to reorder the matrices $\mathbf{S}, \mathbf{T}, \mathbf{U}_{L}$, and $\mathbf{U}_{R}$ such that the vector to be targeted is stored in the first column of $\mathbf{U}_{R}$, denoted by $\mathbf{U}_{R}(:, 1)$, with the corresponding eigenvalue stored in $\mathbf{S}(1,1) / \mathbf{T}(1,1)$. This reordering is not difficult and is discussed in Ref. 19 with an actual implementation given in Ref. 20. The power of the method comes from the fact that the reordering is arbitrary. For targeting eigenvectors in the interior of the spectrum, one would order the matrices according to how close they are to the reference energy or

$$
\left|\varepsilon_{r e f}-\mathbf{S}(1,1) / \mathbf{T}(1,1) \leqslant\right| \varepsilon_{r e f}-\mathbf{S}(2,2) / \mathbf{T}(2,2) \mid \leqslant \ldots .
$$

Another option is to order the matrices based on their localization properties in space, for example, states localized at an interface or at a surface. In principle, any property that can 
be obtained from the eigenvectors can be targeted. Examples are given later demonstrating both eigenvalues and localization property targeting.

Once the matrices have been reordered, the new target vectors can be calculated as

$$
\mathbf{q}=\mathbf{U}_{R}(:, 1), \quad \mathbf{z}=\mathbf{U}_{L}(:, 1) .
$$

From this, the new residual can be calculated to test for convergence as follows:

$$
\mathbf{r}=(\mathbf{T}(1,1) \mathbf{H}-\mathbf{S}(1,1) \mathbf{O}) \mathbf{q} .
$$

If the residual is less than the convergence tolerance, updating the generalized Schur matrices deflates the eigenproblem:

$$
\mathbf{Q} \leftarrow[\mathbf{Q q}], \quad \mathbf{Z} \leftarrow[\mathbf{Z z}] .
$$

One then selects the next vector in the sort to target and the process continues until all eigenvectors are found.

\section{B. Restarting}

The test and search subspaces are not allowed to grow without bound. Instead they are allowed to grow only up to a maximum size $n_{\max }$ before they are shrunk back down to a minimum base $n_{\min }$. This is easily accomplished with

$$
\begin{gathered}
\mathbf{V}_{\text {restart }} \leftarrow \mathbf{V U}_{R}\left(:, 1: n_{\min }\right), \\
\mathbf{W}_{\text {restart }} \leftarrow \mathbf{W U}_{L}\left(:, 1: n_{\min }\right) .
\end{gathered}
$$

Because of the reordering process discussed previously, this allows the most promising vectors to be kept while discarding the rest. Typically the range of $n_{\min }$ is $10-20$ and $n_{\max }$ is $20-40$.

\section{CONVERGENCE AND SCALABILITY}

If the Jacobi-Davidson correction equation is solved exactly, the method seems to have cubic convergence. ${ }^{23}$ However, this is not explicitly proven. Even though the method is designed to target a single eigenvector at a time, one builds up information on nearby eigenvectors. For this reason one would expect that after the first vector is found the remaining vectors would converge at a fairly uniform rate, which is shown to be true in the examples below.

The degenerate and nearly degenerate eigenvalues also do not pose a problem (see examples below). This is mainly due to two reasons. The first is due to deflation of the eigenproblem. With deflation the degenerate eigenvectors found are removed from the spectrum. This keeps eigenvectors already found from interfering with the current target vector. The other reason can be understood by looking at Fig. 1. Notice that if the reference energy is close to a nearly degenerate eigenvector, the shifted and inverted eigenvalue tends to infinity-widely separating it from other nearby nearly degenerate eigenvectors and making the eigenvalue an extremum that is easily targeted. One potential problem with degenerate eigenvalues can occur if the test and search subspaces are not large enough to hold all the degenerate vectors. Since only a single eigenvector is targeted at a time there is the worst-case possibility of selecting a different degenerate vector to target at each iteration. However, if all the degenerate vectors can be held in the search subspace then they would all tend to converge at about the same rate. On the other hand, if the search subspace is too small, then every correction vector added could add information on a vector not contained in the search subspace. The subsequent shrinking of the subspace when it had reached its maximum size would eliminate some of these degenerate vectors causing the process to repeat. In this case, one could always be adding a vector that corresponds to a new degenerate vector.

We now discuss the scalability of the method with system size. We assume in the following that the number of eigenpairs that need to be found is much less than the total number of eigensolutions of $\mathbf{H}$ in a given basis set. The number of eigenpairs needed is typically independent of the number of atoms. Take for example, using spectrum targeting for determining the band gap. In theory one only needs two eigenvectors, the highest occupied eigenstate and lowest unoccupied eigenstate, to determine the band gap. This does not depend on the number of atoms. In practice though one is not guaranteed to find the above states first. Instead one will find several states on both sides of the Fermi surface and from these determine the band gap. In this case, the number of eigenpairs found is still independent of the number of atoms, i.e., if the system size is doubled the total number of states found remains fixed. Although this does correspond to a slightly larger prefactor for the overall scaling.

The work for each iteration scales the same as an individual matrix-vector operation for $\mathbf{H} \psi$ or $\mathbf{O} \psi$. For large systems each $\mathbf{H} \psi$ operation is dominated by the number of nonlocal terms (e.g., in the pseudopotential approach), which scales linearly with the number of atoms. The size of each vector also scales linearly with the number of atoms. For this reason, if a plane-wave basis set is chosen and the nonlocal terms are evaluated in plane-wave space, the algorithm has quadratic scaling or $O\left(n^{2}\right)$ (where $n$ is the number of atoms). On the other hand, if these terms are evaluated in real space they can be performed in parallel by making use of the space locality of these terms. This approach has a scaling of $O\left(n \ln _{2} n\right)$ due to the two fast Fourier transforms to transfer the solution to/from Fourier space. Finally, if all computations are performed in real space, the method scales linearly, $[O(n)]$ with the number of atoms.

\section{EXAMPLES}

The following are the three examples of application of the method for three different materials in the solid state within density-functional theory (DFT). ${ }^{13}$ The examples have been chosen to highlight the different strengths of the method. The desired accuracy in the wave function has been chosen to be $10^{-5}$, i.e.,

$$
\|\mathbf{H} \psi-\varepsilon \mathbf{O} \psi\| \leqslant 10^{-5} .
$$

When the number of basis functions are taken into account this corresponds to a rms accuracy of $10^{-7}$ for each basis function coefficient. Note that this accuracy is considerably 


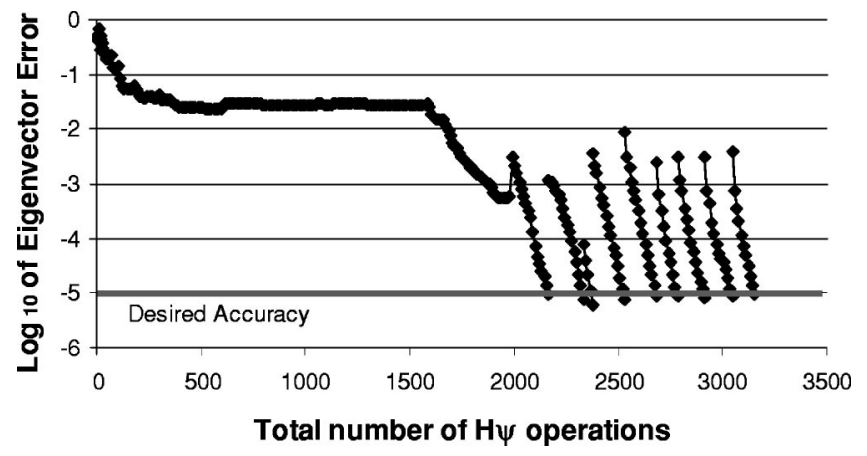

FIG. 2. Convergence for the nine closest eigenvectors to the target energy for an $\operatorname{In}_{64} \mathrm{P}_{64}$ supercell using semiempirical pseudopotentials. The energy chosen was to test the method's ability to handle degenerate and nearly degenerate eigenstates. There are a total of 150 eigenstates within $\pm 0.1 \mathrm{Ry}$ of the reference energy. The pseudopotentials used result in a standard eigenvalue problem.

higher than usually required in the total-energy calculations. The iterative solver GMRES (Ref. 17) has been used with a maximum of 10 iterations allowed per target vector. In all cases, we plot the logarithm of the error in the currently targeted wave function versus the total number of $\mathbf{H} \psi$ operations performed. Deflation has been used to remove the converged eigenvectors so that they are not "found" again as described earlier. No preconditioning was used in the spectrum targeting examples, and only a simple preconditioner was used in the localization targeting example. Use of an appropriate preconditioner can considerably decrease the number of $\mathbf{H} \psi$ operations performed.

\section{A. Spectrum targeting}

The first example is an $\operatorname{In}_{64} \mathrm{P}_{64}$ supercell. We used the semiempirical pseudopotentials obtained from $\mathrm{Fu}$ and Zunger $^{21}$ and an energy cutoff of $32 \mathrm{Ry}$. This is an example of a standard eigenvalue problem, i.e., $\mathbf{O}=\mathbf{I}$, or the identity operator. The reference energy was chosen because of the high number of nearly degenerate eigenstates. There exist over 150 eigenstates within an energy range of $\pm 0.1 \mathrm{Ry}$ around the reference energy. This represents a very challenging problem due to high degeneracy. The nine eigenstates closest to the reference were chosen (see Fig. 2). The test and search subspaces are limited to $n_{\min }=20$ and $n_{\max }=40$. These are much smaller than the degeneracy and could lead to problems as discussed previously. However, in the case at hand we found that this did not occur. Notice that over half of the work goes to building up a good test and search subspace.

The second example is a small $\alpha$-quartz supercell of nine atoms. We used the PAW method with a fixed Hamiltonian to solve the eigenproblem and an energy cutoff of $36 \mathrm{Ry}$. As stated earlier, the PAW method requires solving a generalized eigenvalue problem. The results are shown in Fig. 3 where the ten eigenstates closest to the target were selected. It is evident from Figs. 2 and 3 that either the standard eigenvalue problem or the generalized eigenvalue problem do not pose any difficulty and require a comparable number of $\mathbf{H} \psi$ operations.

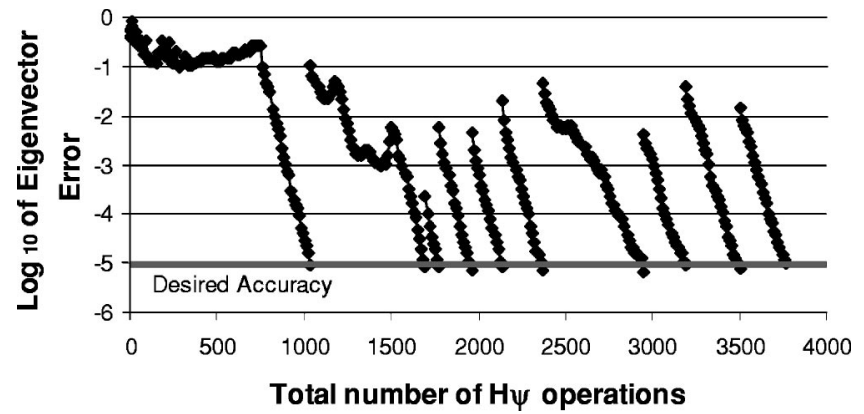

FIG. 3. Example of eigenvalue targeting with a generalized eigenvalue problem. A nine-atom $\mathrm{SiO}_{2}$ supercell was used with a fixed PAW Hamiltonian. The ten eigenstates closest to the reference energy were found.

A considerable improvement with respect to the FS method can be readily seen. As stated previously, the FS method requires on average $\sim 200$ applications of $\mathbf{H}$ per band per iteration. ${ }^{15}$ In general, about 5-10 iterations are required to reach convergence using the FS method. This amounts to about 1000-2000 applications of $\mathbf{H}$ per band. From Figs. 2 and 3, it is evident that we need less than 4000 $\mathbf{H} \psi$ operations for 9 and 10 eigenvalues, respectively, which corresponds to less than 400 applications of $\mathbf{H}$ per band. Furthermore, the example in Fig. 3 represents a generalized eigenvalue problem. As we have stated in Sec. II B, in the FS method the condition number of this problem increases cubically over the original eigenvalue problem and, therefore, would require more than 1000 applications of $\mathbf{H}$ per band per iteration, or, equivalently, more than $10^{4}$ applications of $\mathbf{H}$ per band to reach convergence. On the other hand, as it is evident from Fig. 3, the condition number of the generalized eigenvalue problem is practically unchanged within the present method.

\section{B. Localization targeting}

The last example demonstrates the ability to target localization properties of the eigenvectors by finding the lowestenergy eigenvector situated around each of the $27 \mathrm{Ca}$ atoms in a $\mathrm{Ca}_{27} \mathrm{~F}_{54}$ supercell (see Fig. 4). An energy cutoff of $64 \mathrm{Ry}$ has been used. In the present case, we look for the eigenvectors $\psi$ such that the probability

$$
\sum_{i} \int_{0}^{r_{0}} d \mathbf{r}\left|\psi\left(\mathbf{r}-\mathbf{r}_{i}\right)\right|^{2}
$$

is maximized, where $\mathbf{r}_{i}$ is the position of the $i$ th $\mathrm{Ca}$ atom in the cell, and $\mathbf{r}_{0}$ is a "localization radius," which we assume to be 3 a.u. Again, the PAW method was used, requiring the solution of a generalized eigenvalue problem. It should be noted that the wave functions were not truncated in any way outside the augmentation region. With the use of standard approaches, such a problem would require spanning the whole energy spectrum with subsequent analysis of each individual eigenvector.

A simple preconditioner was used in order to speed convergence. The preconditioner $\mathbf{M}$ simply damps the wave function outside the localization radius. This is similar to the 


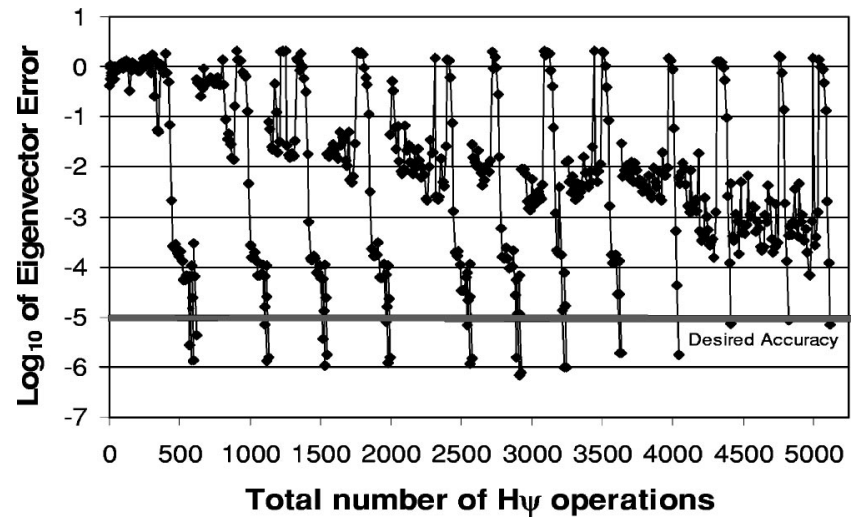

FIG. 4. Space localization example targeting the lowest wave function located on each of the $27 \mathrm{Ca}$ atoms in $\mathrm{Ca}_{27} \mathrm{~F}_{54}$. A fixed PAW Hamiltonian was used resulting in a generalized eigenvalue equation. The simple real-space preconditioner discussed in the text was used.

preconditioner used in Ref. 1 to damp the unwanted large eigenvalue solutions for the standard DFT eigenvalue problem. In the localization targeting case, we are simply damping components of wave functions that exist outside the targeted region. This preconditioner does not, in any way, change the solution. It only affects the convergence rate. It should also be noted that this preconditioner does not attempt to approximate $\mathbf{H}-\varepsilon_{\text {ref }} \mathbf{O}$ as discussed in Ref. 10. The Ref. 10 preconditioner is designed for spectrum targeting only. The preconditioner discussed here is designed for localization targeting only. The form is given below

$$
\mathbf{M x}(\mathbf{r})=\left\{\begin{array}{cc}
\mathbf{x}(\mathbf{r}), & \left|\mathbf{r}-\mathbf{r}_{i}\right| \leqslant r_{0} \\
\frac{3}{4} \mathbf{x}(\mathbf{r}), & \left|\mathbf{r}-\mathbf{r}_{i}\right|>r_{0},
\end{array}\right.
$$

where $\mathbf{x}(\mathbf{r})$ is an arbitrary vector. It can easily be seen that the diagnonal preconditioner defined above does not effect the solution to the original eigenvalue problem. Any solution, $\psi$, to the eigenvalue problem is also a solution to

$$
\mathbf{M}(\mathbf{H} \psi-\varepsilon \mathbf{O} \psi)=0 .
$$

Substituting in the preconditioner definition gives

$$
\begin{gathered}
(\mathbf{H} \psi-\varepsilon \mathbf{O} \psi)=0, \quad\left|\mathbf{r}-\mathbf{r}_{i}\right| \leqslant_{0}, \\
\frac{3}{4}(\mathbf{H} \psi-\varepsilon \mathbf{O} \psi)=0, \quad\left|\mathbf{r}-\mathbf{r}_{i}\right|>r_{0} .
\end{gathered}
$$

In order for the localization targeting to work, one needs to convert the Schur vectors to actual eigenvectors. This is easily done using methods previously discussed.

\section{CONCLUSION}

In conclusion, we have presented an efficient method to target eigenvalues and eigenvectors of a given Hamiltonian using arbitrary selection criteria. The method can easily handle systems with highly degenerate (or quasidegenerate) electronic structures, and does not require the diagonalization of the full Hamiltonian.

Structures with large numbers of atoms can also be easily handled since the method scales linearly with the number of atoms. Finally, the method can easily be extended to generalized eigenvalue problems without an increase in solving difficulty.

\section{ACKNOWLEDGMENTS}

A. Tackett would like to acknowledge Natalie A. W. Holzwarth and G. Eric Matthews at Wake Forest University for numerous helpful discussions, and also for the use of their SP2 where the calculations presented in this paper were carried out. M. Di Ventra acknowledges partial support from the NSF Grant Nos. DMR-01-02277 and DMR-01-33075 and the Carilion Biomedical Institute.
${ }^{1}$ M.C. Payne, M.P. Teter, D.C. Allan, T.A. Arias, and J.D. Joannopoulos, Rev. Mod. Phys. 64, 1045 (1992).

${ }^{2}$ C. Lanczos, J. Res. Natl. Bur. Stand. 45, 255 (1950).

${ }^{3}$ E.R. Davidson, J. Comp. Physiol. 17, 87 (1975).

${ }^{4}$ C.W. Murray, S.C. Racine, and E.R. Davidson, J. Comp. Physiol. 103, 382 (1992).

${ }^{5}$ D.M. Wood and A. Zunger, J. Phys. A 18, 1343 (1985).

${ }^{6}$ L.-W. Wang and A. Zunger, J. Chem. Phys. 100, 2394 (1994).

${ }^{7}$ L.-W. Wang and A. Zunger, Phys. Rev. B 53, 9579 (1996).

${ }^{8}$ A. Franceschetti and A. Zunger, Nature (London) 402, 60 (1999).

${ }^{9}$ G.H. Golub and C.F. Van Loan, Matrix Computations (Johns Hopkins University Press, Baltimore, 1996).

${ }^{10}$ D.R. Fokkema, G.L.G. Sleijpen, and H.A. Van der Vorst, SIAM J. Sci. Comput. (USA) 20, 94 (1998).

${ }^{11}$ P.E. Blöchl, Phys. Rev. B 50, 17953 (1992).

${ }^{12}$ D. Vanderbilt, Phys. Rev. B 41, 7892 (1990).

${ }^{13}$ W. Kohn and L.J. Sham, Phys. Rev. 140, A1133 (1965).

${ }^{14}$ See the introduction in G.L.G. Sleijpen and H.A. Van der Vorst,
SIAM J. Matrix Anal. Appl. 17, 401 (1996) for a more exhaustive list and comparison.

${ }^{15}$ See endnote 10 in Ref. 6.

${ }^{16}$ In the PAW and ultrasoft framework, for instance, this can easily be done by using the Woodbury formula. See, e.g., p. 35, CRC Standard Mathematical Tables, 26th ed. (CRC Press, Boca Raton, 1981).

${ }^{17}$ Y. Saad and M.H. Schultz, SIAM (Soc. Ind. Appl. Math.) J. Sci. Stat. Comput. 7, 856 (1986).

${ }^{18}$ G.L.G. Sleijpen and H.A. Van der Vorst, Elec. Trans. Numer. Anal. 1, 11 (1993).

${ }^{19}$ P. Van Dooren, SIAM (Soc. Ind. Appl. Math.) J. Sci. Stat. Comput. 2, 121 (1981).

${ }^{20}$ P. Van Dooren, ACM Trans. Math. Softw. 8, 376 (1982).

${ }^{21}$ H. Fu and A. Zunger, Phys. Rev. B 55, 1642 (1997).

${ }^{22}$ J.P. Killingbeck and G. Jolicard, Chem. Phys. Lasers 255, 79 (1996).

${ }^{23}$ G.L.G. Sleijpen and H.A. Van der Vorst, SIAM J. Matrix Anal. Appl. 17, 401 (1996). 